\title{
Title: Establishing a measurement array to assess tissue tolerance during loading representative of prosthetic use
}

\author{
J. L. Bramley ${ }^{1}$, P. R. Worsley ${ }^{2}$, L.E. Bostan ${ }^{2}$, D. L. Bader ${ }^{2}$, A. S. Dickinson ${ }^{1}$ \\ ${ }^{1}$ Bioengineering Science Research Group, Faculty of Engineering and Physical Sciences, University \\ of Southampton, UK; and \\ ${ }^{2}$ Skin Health Research Group, Faculty of Environmental and Life Sciences, University of \\ Southampton, UK;
}

\begin{abstract}
:
Background: In the early stages of rehabilitation after primary amputation, residual limb soft tissues have not been mechanically conditioned to support load and are vulnerable to damage from prosthetic use. There is limited quantitative knowledge of skin and soft tissue response to prosthetic loading.
\end{abstract}

Methods: An in-vivo protocol was developed to establish suitable measures to assess tissue tolerance during loading representative of early prosthesis use. Ten participants without amputation were recruited, and one participant with trans-tibial amputation, with pressure applied to their calf in increments from 20 to $60 \mathrm{mmHg}$. Measurements were recorded at relevant skin sites, including interface pressures, transcutaneous oxygen $\left(\mathrm{T}_{\mathrm{C}} \mathrm{PO}_{2}\right)$ and carbon dioxide $\left(\mathrm{T}_{\mathrm{C}} \mathrm{PCO}_{2}\right)$ tensions and the inflammatory biomarkers.

Findings: At the maximum cuff pressure, mean interface pressures were between $66-74 \mathrm{mmHg}$, associated with decreased $\mathrm{T}_{\mathrm{C}} \mathrm{PO}_{2}$ values. On the release of pressure, the ischaemic response was reversed. Significant upregulation $(\mathrm{p}<0.05)$ in an inflammatory biomarker, IL-1 $\alpha$, and its antagonist, IL-1RA, were observed at all sites immediately following loading.

Interpretation: The protocol was successful in applying representative prosthetic loads to lower limb tissues and monitoring the physiological response, both in terms of tissue ischemia and skin inflammation. Results indicated that the measurement approaches were sensitive to changes in interface conditions, offering a promising approach to monitor tissue status for people with amputation.

\section{Keywords:}

amputation; pressure; tissue damage; ischaemia; inflammatory response; inflammation biomarker.

Correspondence: A. S. Dickinson, Bioengineering, Faculty of Engineering and Physical Sciences, University of Southampton, Highfield Campus, University Rd, Southampton, SO17 1BJ, UK, (Email: alex.dickinson@ @oton.ac.uk) 


\section{Introduction}

There are many situations where skin and soft tissues are compromised by mechanical loading from medical devices, which interface with the body. The tissue deformations resulting from interface pressures and shear forces may cause damage, in the form of pressure ulcers (PUs). There are two main damage mechanisms, namely:

(i) direct cell deformation where the internal mechanical conditions generated within the tissues lead to a loss of cell membrane integrity [1], and

(ii) prolonged tissue deformation which results in occlusion of blood and lymphatic vessels leading to compromised transport of oxygen-carrying blood cells and accumulation of waste products and toxins, respectively $[2,3]$.

The reperfusion coincident with periods of off-loading can result in further tissue damage through the release of cytotoxic reactive oxygen species (ROS), inflammation and the recruitment of white blood cells [4].

One example of tissue compromise resulting from medical device interaction involves individuals with lower limb amputation. Soft tissue discomfort and damage has a reported prevalence of $36-66 \%$ [5], with scar tissue representing areas of particular vulnerability [6]. The residual limb tissues form a critical loaded interface with the prosthetic socket during activities of daily living (ADL). Tissue loading will be influenced by the socket design, which is produced by a prosthetist who considers both the morphology of the tissues and their ability to tolerate load [7-9]. A congruent coupling is desirable to avoid in-socket motion and instability. Accordingly, both oversized and undersized sockets can lead to soft tissue damage, reduced function and discomfort [9]. Critically, in the early stages post-primary-amputation the reconstructed soft tissues are not conditioned to support load, and therefore are vulnerable to tissue damage, often termed stump ulcers. Clearly, its development will depend upon a number of factors associated with the individual and the biomechanical adaptation in response to loading with a prosthesis $[10,11]$. Common comorbidities associated with amputation, such as vascular disease, will certainly increase the susceptibility to tissue damage due to impaired perfusion and sensory perception [12]. In addition, the interface between the limb and the socket typically exhibits an elevated temperature and humidity, each of which will reduce the tissue tolerance to loading and increase friction at the interface $[13,14]$.

The interface conditions between the residuum and the socket have been characterised using a variety of sensor arrays, based on various physical principles [15-17]. Typical interface values measured during static prosthetic weight bearing range considerably from 0.5 to $125 \mathrm{kPa}$ (4 to $938 \mathrm{mmHg}$ ) and 1 to $52 \mathrm{kPa}$ ( 8 to $389 \mathrm{mmHg}$ ) for pressure and shear stress, respectively [15-17]. This is largely dependent on individual socket designs and sensor characteristics. However, interface measurements will not necessarily correspond to either internal conditions of the residuum soft tissues or their physiological response. To address this, various strategies have been used to monitor the status of loaded dermal tissues [18]. Transcutaneous gas tensions $\left(\mathrm{T}_{\mathrm{C}} \mathrm{PO}_{2} / \mathrm{T}_{\mathrm{C}} \mathrm{PCO}_{2}\right)$ have been employed as a measure of local tissue ischaemia in response to different loading conditions with reference to indenters [19], the residuum-socket/liner interface [20,21] and various designs of support surfaces $[22,23]$. Such studies have revealed distinct categorical responses in terms of oxygen and carbon dioxide gas tensions relative to the loading regimens [24]. Biomarkers have also been used to assess the physiological reaction to mechanical loads. As an example Interleukin-1 $\alpha$ (IL-1 $\alpha$ ), and its competitive inhibitor Interleukin-1 Receptor Antagonist (IL-1RA), are inflammatory cytokines released in response to cell deformation or damage and represent precursors to cell death [25, 26]. IL$1 \alpha$, collected in sebum at the skin surface, has provided a non-invasive measurement technique which 
is sensitive to loads applied to dermal tissues [27-29]. To date, some of these techniques have been used in isolation to assess tissues subjected to prosthetic loading. However, there is a need to develop an array of measurements to assess both the biomechanical and physiological response of soft tissues under loading experienced at the residuum-socket interface.

The present study has been designed to establish a measurement protocol that will be suitable to assess an array of dermal tissue tolerance markers during representative prosthetic loading. This will be achieved by establishing a series of measurements including interface pressures, transcutaneous gas tensions and biomarker response at relevant tissue sites before, during and after loading representative of prosthetic use. The presented protocol has been applied to a cohort of able-bodied participants, and one participant with trans-tibial amputation, prior to further assessment of individuals with amputation.

\section{Materials and Methods}

\subsection{Study Design}

An observational study was conducted on a cohort of consenting able-bodied participants, without amputation, and one participant with trans-tibial amputation. Participants were included if they were over 18 years old, in good health and had no history of skin-related conditions or neurological or vascular pathologies. They also had to be able to remain seated for approximately two hours. Ethics Committee approval for this protocol was granted by the University of Southampton (ERGO IDs: 29696 and 41864).

\subsection{Material and Methods}

A pressure cuff (Ref 0124 Aneroid Sphygmomanometer, Bosch + Sohn GmbH, Germany) was applied over the right calf of each participant and both residual and contralateral limbs of the participant with amputation (Figure 1). A Prosthetic liner (ContexGel Liner, RSL Steeper, UK) was positioned underneath the cuff to provide a representative material to interface with the skin. Three 50 x $50 \mathrm{~mm}$ sites were selected for measurement on each limb: the patellar tendon (above and located centrally with the tibial tuberosity), the lateral calf (just below and forward of the fibula head) and positioned at the same height centrally on the posterior calf (Figure 1A) [11]. These sites were selected as they represent distinct anatomical regions, which are often considered to be pressuretolerant and thus suitable for load transfer when prosthetists create sockets. Hair was removed at the measurement sites by shaving at least 48 hours prior to testing to avoid an upregulation of proinflammatory cytokine due to its associated mechanical irritation [30]. Throughout testing participants were in a seated position on a standard hospital bed with adjustable backrest. Transcutaneous gas tensions were monitored (see below) for a 20 minute unloaded period, in order to estimate baseline gas tension values prior to cuff application. After cuff application the gas tensions were collected for 10 minutes with no inflation pressure. The cuff was then inflated by $10 \mathrm{mmHg}$ increments every 10 minutes in order to achieve pressures ranging from 20 to $60 \mathrm{mmHg}$ (Figure 1C). The reported pressure gauge accuracy was $\pm 3 \mathrm{mmHg}$ [31]. These pressure magnitudes represent those commonly experienced during early prosthetic rehabilitation using the Pneumatic Post-Amputation Mobility (PPAM) Aid [32]. Comparable studies have applied similar pressures over this duration safely with pressures reported to have an effect on the vascular and lymphatic supply of local tissues [22, 33, 34]. The pressures were subsequently released, by $10 \mathrm{mmHg}$ increments every 30 seconds, to a zero inflation pressure, which was maintained for a 40 minute refractory period (Figure 1C). 
A

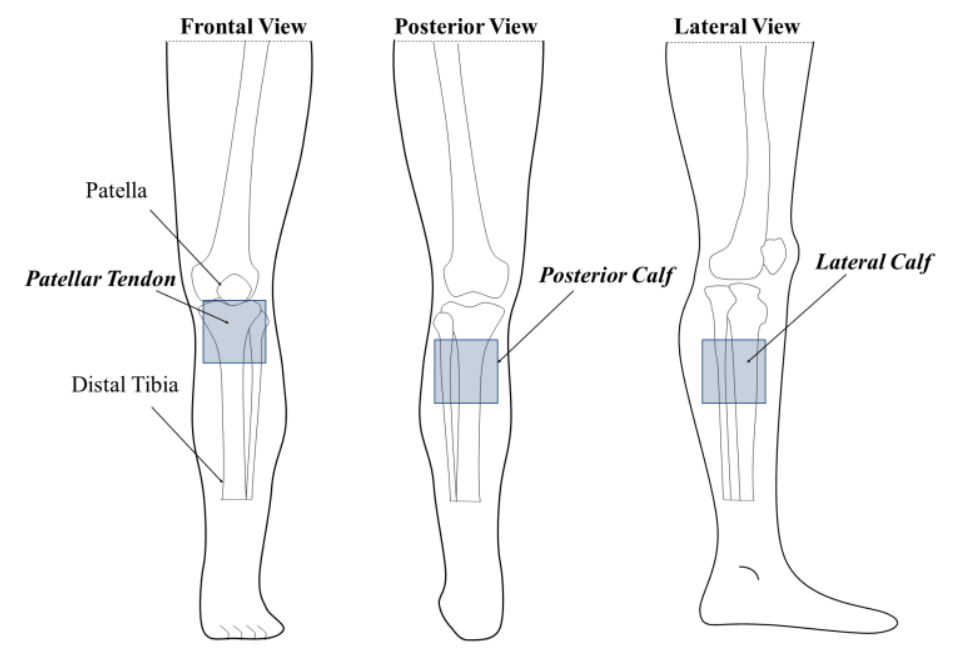

B

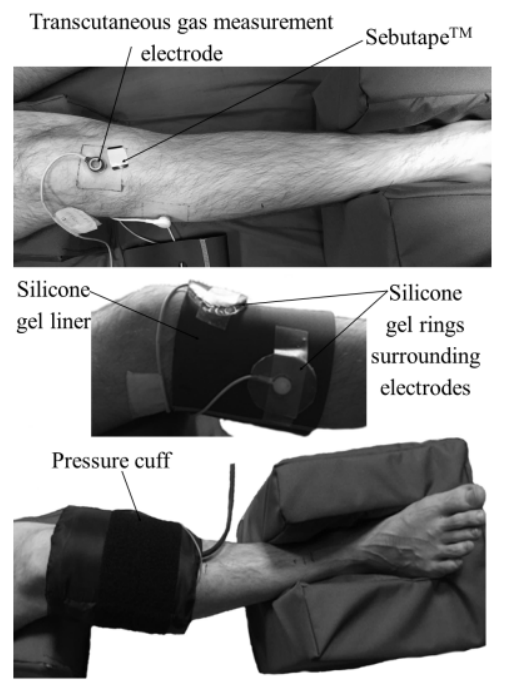

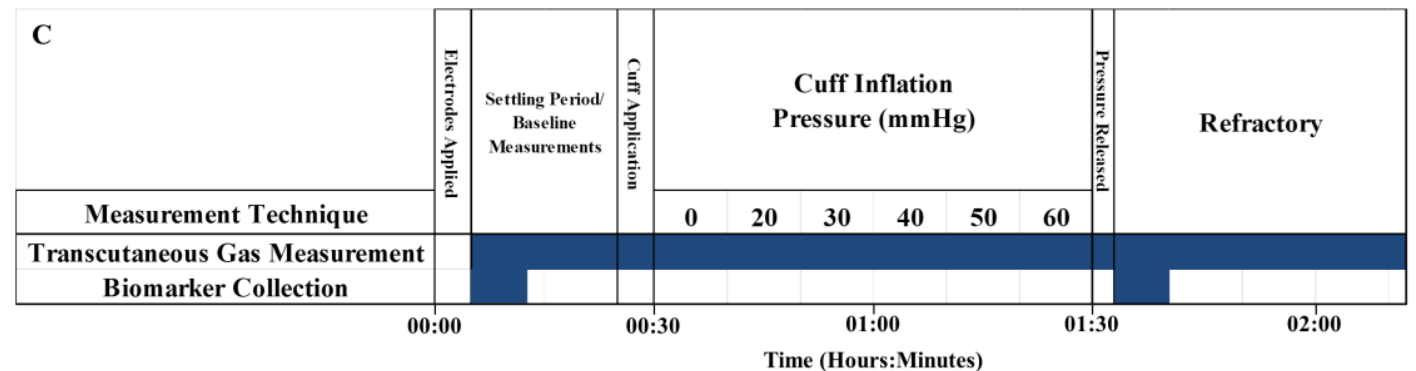

Figure 1 A: Measurement sites each of area 50 x $50 \mathrm{~mm}$, on the right lower limb, B: Anterior view of transcutaneous electrode and Sebutape positioned at the patellar tendon measurement site(top), side view of silicone liner around all three measurement sites and silicone gel rings positioned around the transcutaneous electrode at each measurement site (middle) and pressure cuff wrapped around the three measurement sites with the participants' limb in the supported testing position (bottom), C: Schematic representing the test session protocol

Interface pressures were measured using an pneumatic pressure monitoring system (Mk III, Talley Medical, Romsey, UK) incorporating $28 \mathrm{~mm}$ diameter cells, with a reported mean error of $12 \pm 1 \%$ and a repeatability of $\pm 0.53 \mathrm{mmHg}$ [35]. One cell was used at each measurement site positioned between the skin and TCM electrode. Measurements were recorded after fluctuations in pressures settled to less than $3 \mathrm{mmHg}$, which was after approximately 10 seconds. Transcutaneous oxygen $\left(\mathrm{T}_{\mathrm{C}} \mathrm{PO}_{2}\right)$ and carbon dioxide $\left(\mathrm{T}_{\mathrm{C}} \mathrm{PCO}_{2}\right)$ tensions were monitored at the patellar tendon using combined electrodes (E5280 and TCM5). $\mathrm{T}_{\mathrm{C}} \mathrm{PO}_{2}$ was monitored at the lateral and posterior calf using single channel electrodes (E5250), attached to separate monitors (TCM4, TCM400 Radiometer, Denmark) sampling at a frequency of $0.5 \mathrm{~Hz}$. The patellar tendon was selected for the combined $\mathrm{T}_{\mathrm{C}} \mathrm{PO}_{2}$ and $\mathrm{T}_{\mathrm{C}} \mathrm{PCO}_{2}$ measurement as it represents a site commonly selected for prosthetic load bearing [36]. Rings of silicone gel, representative of the liner thickness, were used to minimise pressure gradients at the electrode-skin interface (Figure 1A).

A sebum sample was collected both prior to and following the incremental loading from the bottom right corner of each $50 \times 50 \mathrm{~mm}$ measurement site by applying adhesive tape (Sebutape ${ }^{\mathrm{TM}} \mathrm{CuDerm}$, Dallas, TX, USA) at baseline prior to cuff application and post-loading immediately after cuff removal, using a standardised protocol [37]. Although Perkins et al utilised a 1 minute sampling period [37], in recent research 2 minute sampling periods have been used [27-29, 38-40] to provide sufficient time to sample the biofluid, namely sebum. Sebutapes were applied to the skin using 
tweezers and a roller to avoid cross-contamination, and remained in-situ for a period of two minutes. Once removed they were stored in tubes at $-80{ }^{\circ} \mathrm{C}$ prior to biochemical analysis. The frozen tapes were thawed to room temperature and $1.7 \mathrm{ml}$ of phosphate buffered saline (PBS; Sigma-Aldrich Co, St. Louis, Missouri, USA) with 0.05\% TWEEN solution (Sigma-Aldrich Co, St. Louis, Missouri, USA) was added to each tube to recover proteins. After immersion for one hour, the tubes were sonicated for 10 minutes in a room temperature water bath, and then vortexed vigorously for two minutes. The concentrations of pro-inflammatory cytokines, IL-1 $\alpha$ and IL-1RA, were estimated using pre-coated ELISA analysis plates (Meso Scale Diagnostics, USA) [37]. The total protein (TP) on each tape was also estimated using a protein assay kit (Thermo Fisher Scientific, UK).

\subsection{Data Analysis}

Raw data from each measurement technique were processed and analysed using MATLAB (Mathworks, USA) and SPSS Statistics (IBM, USA). Transcutaneous gas data were analysed to evaluate the degree of ischaemia [24]. Briefly, the percentage changes in $\mathrm{T}_{\mathrm{C}} \mathrm{PO}_{2}$ and $\mathrm{T}_{\mathrm{C}} \mathrm{PCO}_{2}$ from baseline during each loading condition were calculated. At the patellar tendon test site these percentage changes were subsequently categorised into three distinct responses [24]:

- Category 1 (minimal changes in $\mathrm{T}_{\mathrm{C}} \mathrm{PO}_{2}$ and $\mathrm{T}_{\mathrm{C}} \mathrm{PCO}_{2}$ ),

- Category $2\left(>25 \%\right.$ decrease in $\mathrm{T}_{\mathrm{C}} \mathrm{PO}_{2}$ with minimal change in $\left.\mathrm{T}_{\mathrm{C}} \mathrm{PCO}_{2}\right)$ and

- Category $3\left(>25 \%\right.$ decrease in $\mathrm{T}_{\mathrm{C}} \mathrm{PO}_{2}$ and $>25 \%$ increase in $\left.\mathrm{T}_{\mathrm{C}} \mathrm{PCO}_{2}\right)$.

A Category 3 response is indicative of compromised tissue viability [23]. Ratios of IL-1 $\alpha / \mathrm{TP}$ and IL1RA/TP were calculated to account for intra-participant variation of proteins [27], and presented as the percentage change from the baseline at each measurement site for each participant.

The effect of cuff loads was then investigated using a combination of tests to assess associations between measures and within-individual changes during each test condition. All data were examined for normal distribution prior to analysis using the Shapiro-Wilk test, in order to determine appropriate descriptive and inferential statistics. Pearson's correlation was used to assess the relationship between applied cuff pressure and interface pressure at each test site. In the case of the inflammatory response to applied loading, the data were not normally distributed and thus the non-parametric paired Wilcoxon Signed Rank Test was used. Differences were considered to be statistically significant at the $5 \%$ level $(\mathrm{p}<0.05)$. 


\section{Results}

A cohort of ten participants without amputation was recruited for this study with a mean age of 28 years (range 23 to 36 years), 6 male and 4 female. The mean \pm standard deviation height and weight were $175.9 \pm 9.5 \mathrm{~cm}$ and $69.7 \pm 11.8 \mathrm{~kg}$, respectively. The corresponding body mass index (BMI) was $22.5 \pm 3.4 \mathrm{~kg} / \mathrm{m}^{2}$. Ambient temperature and humidity in the testing laboratory ranged from 22.0 to $24.3^{\circ} \mathrm{C}$ and 31.0 to $45.1 \%$, respectively.

\subsection{Interface Pressure}

At a cuff inflation pressure of $60 \mathrm{mmHg}$, the mean interface pressures ranged from 66 to $74 \mathrm{mmHg}$, with the highest values generally occurring at the patellar tendon (Figure 2). There was a clear monotonic increase in interface pressures with cuff inflation pressure at all three tests sites, and this correlation was statistically significant $(r>0.93, \mathrm{p}<0.001)$. Before the cuff was inflated $(0 \mathrm{mmHg})$, finite interface pressures indicated preloading due to the mass of the pressure cuff and its uninflated tension. The pressure cuff was wrapped around the limb and secured in position using Velcro, causing low interface pressures, most notably at the patellar tendon site, an area with low soft tissue coverage over the underlying bony anatomy.

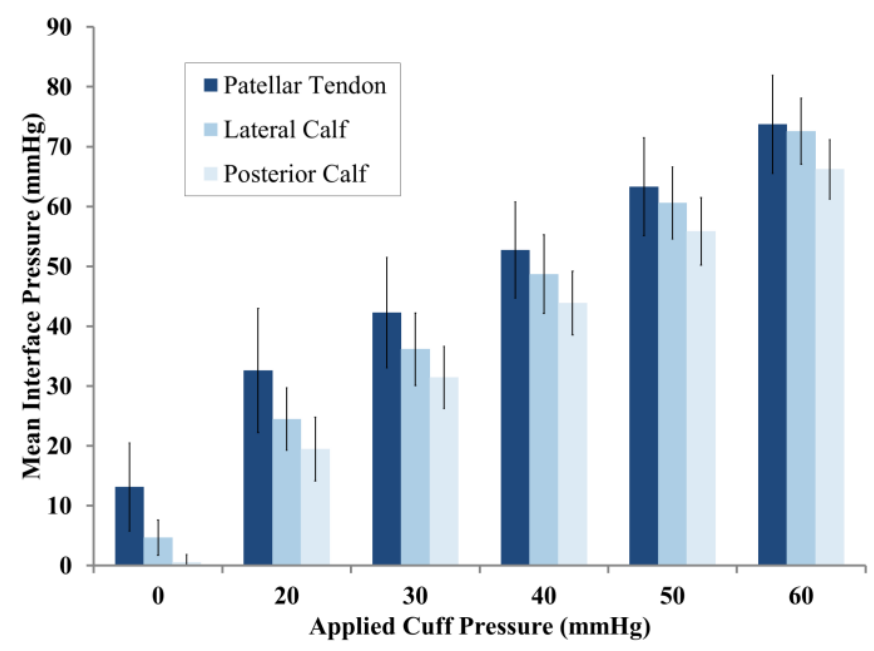

Figure 2: The effects of cuff pressures, applied incrementally to the lower limbs of 10 participants, on mean ( \pm standard deviation) interface pressures at three measurement sites 


\subsection{Transcutaneous Oxygen and Carbon Dioxide}

A decrease in $\mathrm{T}_{\mathrm{C}} \mathrm{PO}_{2}$ was observed with increasing cuff inflation at all measurement sites (Figure 3 ). The patellar tendon site exhibited two distinct trends in the cohort. The majority of participants displayed a Category 3 response [24], with decreasing $\mathrm{T}_{\mathrm{C}} \mathrm{PO}_{2}$ at elevated cuff pressure associated with $>25 \%$ increase in $\mathrm{T}_{\mathrm{C}} \mathrm{PCO}_{2}$ above baseline levels (Figure $3 \mathrm{~A}$ ). By contrast, two participants only demonstrated a Category 2 response, with minimal changes in $\mathrm{T}_{\mathrm{C}} \mathrm{PCO}_{2}(<25 \%)$ despite a reduction in $\mathrm{T}_{\mathrm{C}} \mathrm{PO}_{2}$ (Figure 3B).

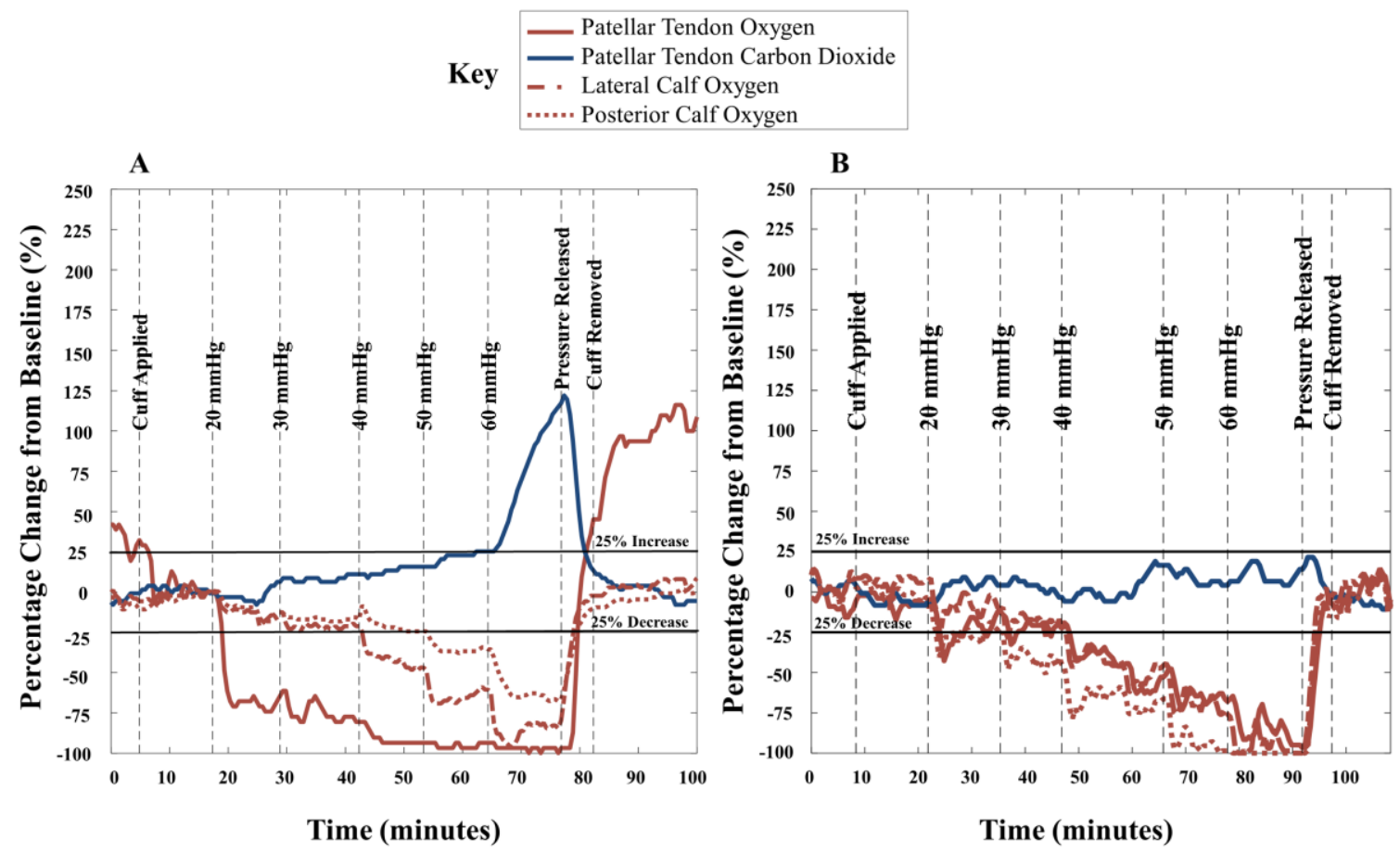

Figure 3: Exemplar participant data showing percentage change from baseline transcutaneous oxygen and carbon dioxide measurements under incremental cuff pressures from 20 to $60 \mathrm{mmHg}$ revealing at the patellar tendon site $A$ :

Category 3 response representative of 8/10 participants and B: Category 2 response representative of 2/10 participants 
Intra-participant variation in ischaemic response at the patellar tendon indicated differences in tolerance to the applied loads. For example, with a cuff pressure of $20 \mathrm{mmHg}$ only one participant exhibited a Category 3 response at the patellar tendon, whereas at $60 \mathrm{mmHg}$ eight participants exhibited a Category 3 response (Figure 4).

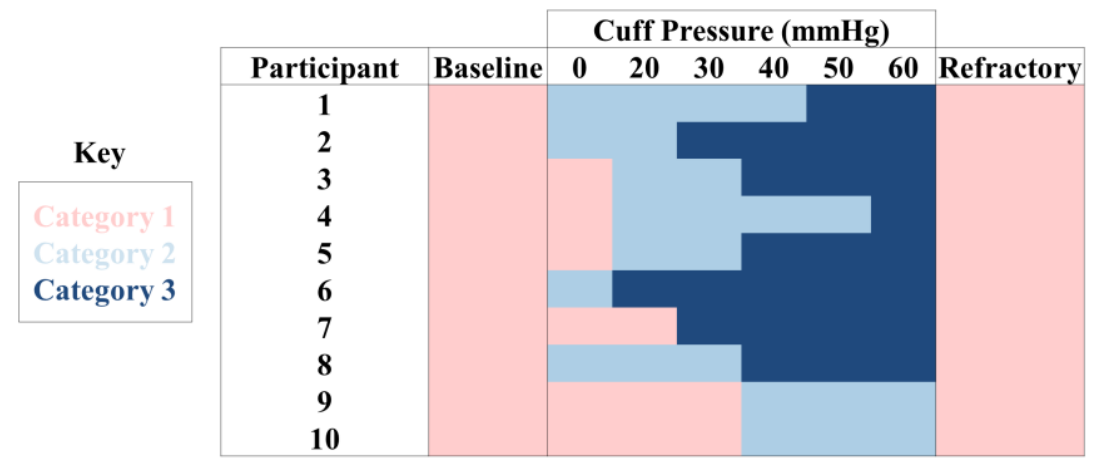

Figure 4: Ischaemic response at the patellar tendon to incremental cuff pressures using categorical analysis [24], which indicates varying tolerance to load across the intact participant group.

With respect to the data from the cohort, there was a mean percentage decrease in $\mathrm{T}_{\mathrm{C}} \mathrm{PO}_{2}$ with incremental cuff pressures (Figure 5), and this correlation was statistically significant $(r>0.74, p<$ 0.001 ). Some saturation was observed when the pressures exceeded $50 \mathrm{mmHg}$ and immediately following load removal all values recovered back to baseline. Each skin site exhibited similar trends with cuff pressures conditions. The greatest $\mathrm{T}_{\mathrm{C}} \mathrm{PO}_{2}$ decrease was observed at the patellar tendon at lower cuff pressures, and at the lateral calf site at higher cuff pressures.

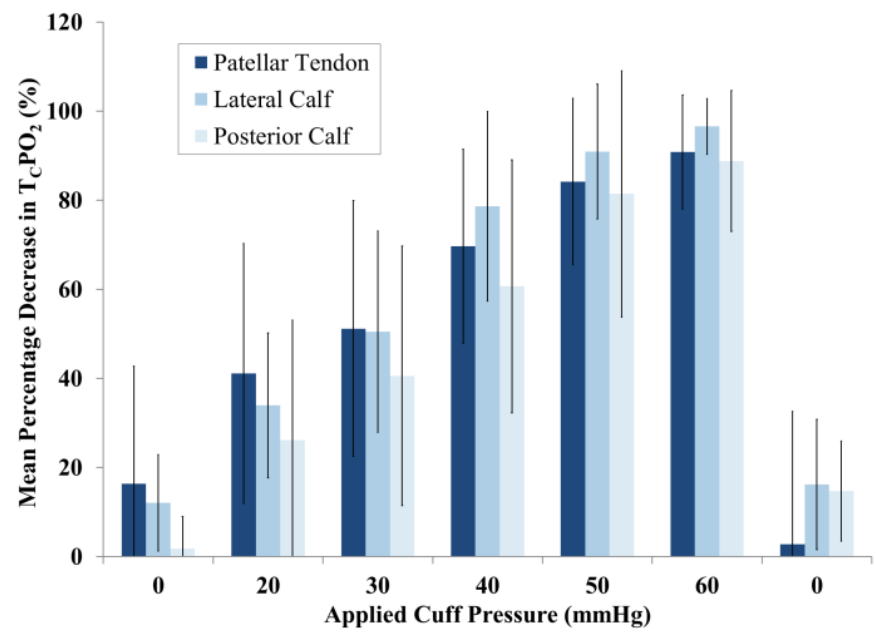

Figure 5: The effects of cuff pressures, applied incrementally to the intact lower limbs of 10 participants, on mean percentage decrease in $\mathrm{T}_{\mathrm{CPO}} \mathrm{PO}_{2}( \pm$ standard deviation) at three measurement sites 


\subsection{Inflammatory Biomarker Analysis}

The cumulative effect from the incremental loading regime resulted in a variable increase in IL-1a/TP and IL-1RA/TP ratio values, reaching statistical significance at patellar tendon and lateral calf sites for both and posterior calf for IL- $1 \alpha / \mathrm{TP}(\mathrm{p}<0.05)$ (Figure 6). The highest upregulation was observed in the patella tendon site, representing a 2 fold increase ( 100\%) from baseline levels.

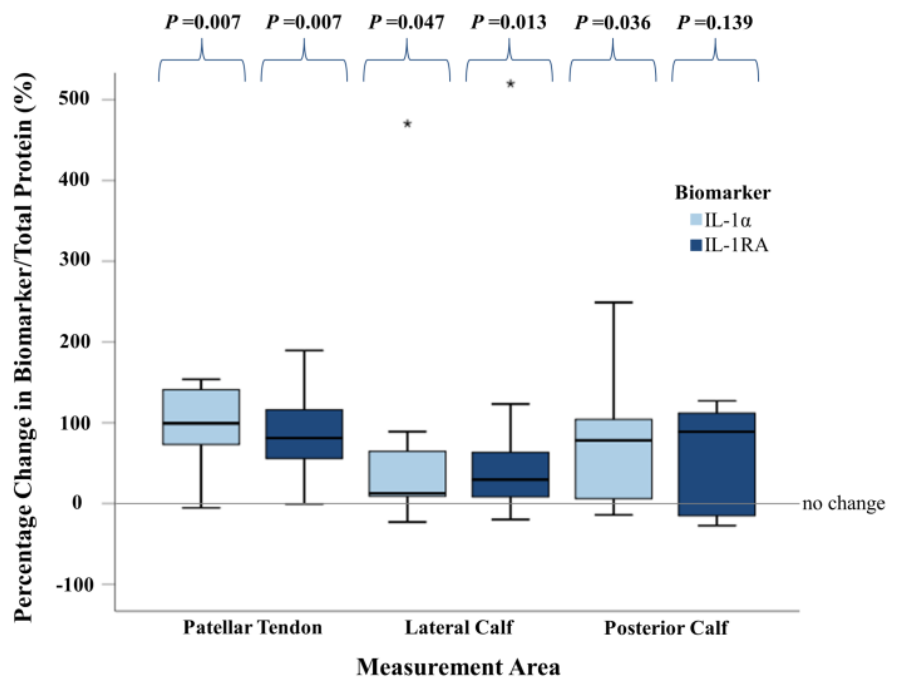

Figure 6: Box and whisker plot showing IL-1 $\alpha /$ Total Protein and IL-1RA/Total Protein ratios, at three measurement sites on 10 healthy participants, expressed as the percentage change between cuff loading at $60 \mathrm{mmHg}$ and baseline,

Note: p-values show the significance of the percentage change in biomarker/total protein from baseline to postloading at each of the measurement sites

On inspection of the individual responses, there was a pre- to post-loading increase in IL-1 $\alpha / \mathrm{TP}$ in the majority of cases ( $9 / 10$ for each site), although there was considerable variation in response magnitude between individuals and within measurement sites (Figure 6 and Appendix Figure 8 for individual participant results). For example in one participant (male, aged 23), IL-1 $\alpha /$ TP more than doubled at both the lateral and posterior calf sites, although minimal change was observed at the patellar tendon. Nevertheless, for the vast majority of participants $(9 / 10)$, there was an upregulation in IL- $1 \alpha / \mathrm{TP}$ ratio in excess of $50 \%$ at the patellar tendon. This response was less consistent at the lateral and posterior calf sites. There was a comparable upregulation in IL-1RA and similar, highly variable inter- and intra-participant trends were observed.

\subsection{Participant with Amputation Example}

A male participant aged 29 with unilateral trans-tibial amputation was recruited for this study to demonstrate the use of the array and protocol in a participant with amputation. His height, weight and corresponding BMI were $165 \mathrm{~cm}, 80 \mathrm{~kg}$ and $29.4 \mathrm{~kg} / \mathrm{m}^{2}$, respectively. His amputation followed recurrent infection after trauma while serving in the military. He was one year post-amputation and an active prosthesis user, reporting 12 to 14 hours usage per day.

As observed in the cohort without amputation, there was a clear monotonic increase in interface pressures with cuff inflation pressure at all three tests sites (Figure 7A). At a cuff inflation pressure of $60 \mathrm{mmHg}$, a Category 2 ischaemic response was observed at the residual limb patellar tendon and a Category 3 response was observed at the contralateral limb patellar tendon (Figures 7C \& D). The cumulative effect from the incremental loading resulted in an upregulation of IL-1 $\alpha$ and IL-1RA, comparable to that observed in the cohort of participants without amputation (Figure 7B). The inflammatory response was greatest at the contralateral limb posterior site. 
A
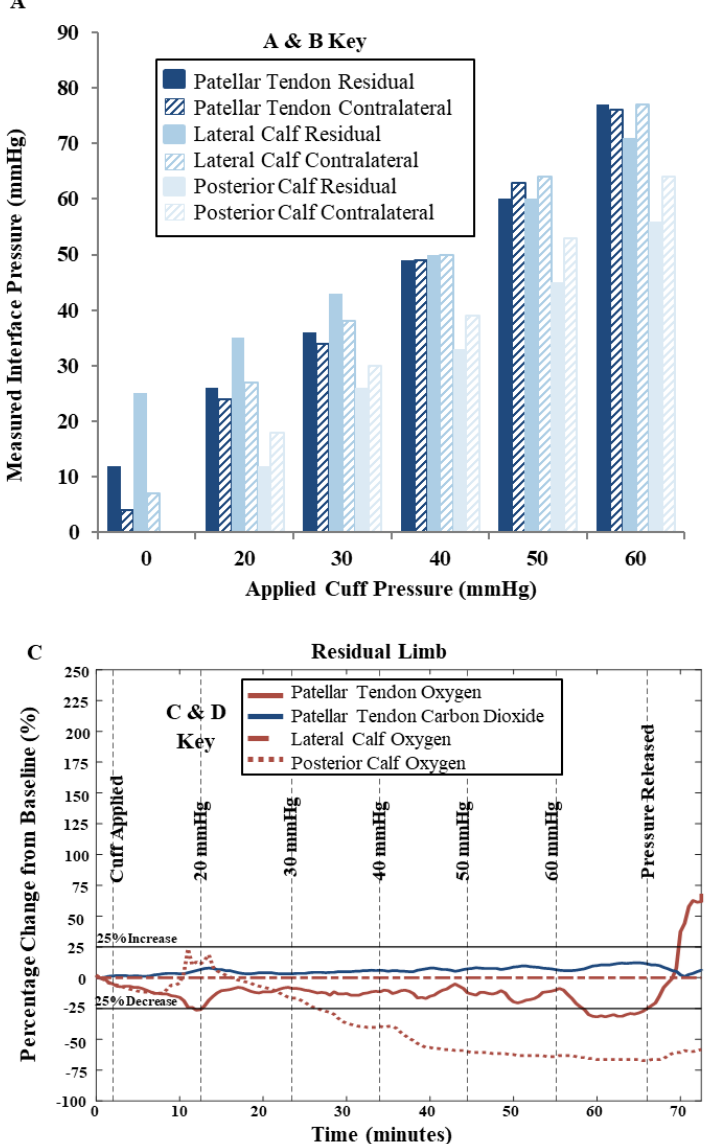
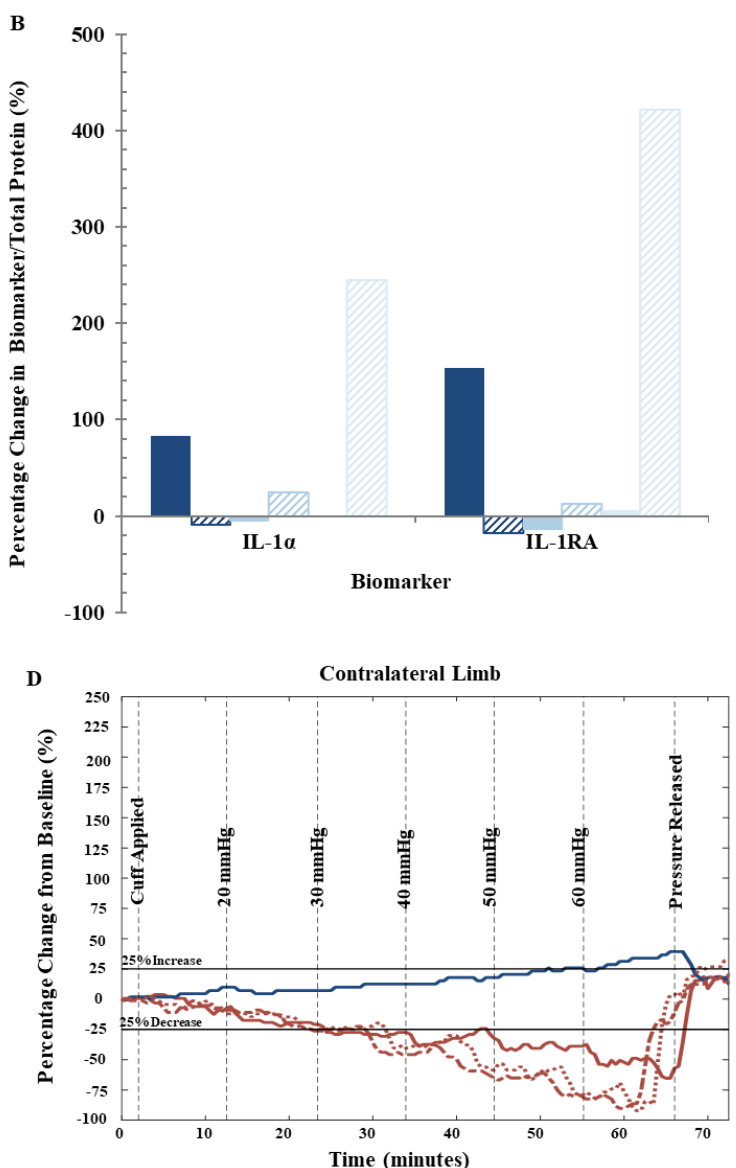

Figure 7: Data from a participant with unilateral trans-tibial amputation including, A: Interface pressures at three measurement sites, estimated at each cuff increment, B: Percentage change in Biomarkers/Total protein ratios from baseline to post-cuff-loading up to $60 \mathrm{mmHg}$ in both limbs at three measurement sites and C \& D: Percentage change from baseline transcutaneous gas tensions at the patellar tendon (oxygen and carbon dioxide) and the lateral and posterior calf sites (oxygen only) under incremental cuff pressures for both limbs. 


\section{Discussion}

This study was designed to establish a measurement array and protocol that could be utilised to assess tissue tolerance at the residual limb-socket interface and elicit further understanding of how soft tissues respond during prosthetic rehabilitation. A cohort of participants without amputation was examined under representative static prosthetic loading to determine whether the proposed measurements could detect changes in local tissue physiology. In order to replicate prosthetic loading using a static PPAM Aid [32], a cuff was inflated incrementally around the lower limb up to $60 \mathrm{mmHg}$. The pressures were observed to compromise tissue viability temporarily, resulting in local ischaemia and an upregulation of inflammatory biomarkers. This demonstrated the potential of the protocol to distinguish between unloaded and loaded conditions and identify critical thresholds whereby tissues were compromised. The protocol was also observed to detect similar tissue changes in both limbs of an age-matched and active person, one year post unilateral trans-tibial amputation. This demonstrates the efficacy of the protocol, although in order to evaluate its full potential for establishing tolerance to prosthetic loading, additional studies are required on individuals with primary and established amputation.

Cuff pressure was found to correlate with interface pressure for each of the three test sites, with the highest values being recorded at the patellar tendon, the site with the thinnest soft tissue coverage (Figure 2). Posterior and lateral calf sites had a slightly higher increase in interface pressure from baseline after applying $60 \mathrm{mmHg}$, potentially due to muscle contractions of participants; however the difference was small compared to the reported error of the Talley pressure sensors $(12 \pm 1 \%)$ [35]. Pressure cuff loading occluded vessels by applying an approximately hydrostatic pressure on the limb. Although not representative of a more focally-rectified socket, this loading technique is more characteristic of a total surface bearing socket or rehabilitation device. In particular, the PPAM aid has been estimated to apply interface pressures at the distal residuum ranging from 4 to $95 \mathrm{mmHg}$ (median $26 \mathrm{mmHg}$ ) and 21 to $125 \mathrm{mmHg}$ (median $28 \mathrm{mmHg}$ ) during standing and walking, respectively [32]. In the test setup, the presence of the transcutaneous gas electrodes produced an effective indentation, causing local internal shear strain and enhancing the study's representation of rectified socket designs.

Ischaemic trends were observed at the patellar tendon, where a Category 3 response was measured in 8/10 participants (Figure 3). It has been reported that elevated and sustained carbon dioxide levels may prove a strong indicator of cell damage through the accumulation of associated anaerobic metabolites and local changes in $\mathrm{pH}$ [41]. There was no discernible demographic reason for the two participants that demonstrated a Category 2 response. The participant with amputation demonstrated a Category 2 response in the residual limb and a Category 3 response in the contralateral limb (Figure 7C \& D), suggesting that the residual limb patellar tendon site may be more tolerant to loading. A larger cohort study could examine whether this is an indication of an enhanced biomechanical adaptation to prosthetic loading. At lower cuff pressures a number of participants showed partial recovery in $\mathrm{T}_{\mathrm{C}} \mathrm{PO}_{2}$ between loading increments, demonstrating some vascular adaptation to load [42] (Figure 3B). However, at $50 \mathrm{mmHg}$ cuff pressure, $\mathrm{T}_{\mathrm{C}} \mathrm{PO}_{2}$ reduced by $>75 \%$ for $8 / 10$ individuals at the patellar tendon and lateral calf and 7/10 at the posterior calf (Figure 5). Equivalent responses have been observed using indenters and liner application on calf tissues [19, 21]. It is of note, that clinical study tissue sites which yielded $\mathrm{T}_{\mathrm{C}} \mathrm{PO}_{2}$ levels below approximately $35 \mathrm{mmHg}$ were observed to be at risk of poor healing post-amputation [43, 44]. In the present study, the majority of participants demonstrated baseline $\mathrm{T}_{\mathrm{C}} \mathrm{PO}_{2}$ higher than this $35 \mathrm{mmHg}$ threshold. The present study does indicate that establishing standard thresholds for tissue health may prove problematic due to the large variability in transcutaneous responses, even in the healthy cohort under investigation (Figure 4). This 
diverse response within able-bodied participants has also been observed in previous studies [23, 24]. In all cases, tissue sites demonstrated a rapid recovery in transcutaneous gas tensions following cuff, pressure release, suggesting complete reperfusion and restoration of tissue health.

IL-1 $\alpha /$ TP increased significantly post-loading with a median percentage change of $99 \%, 19 \%$ and $78 \%$ at the patellar tendon, lateral calf and posterior calf respectively (Figure 6). Similar changes were observed in a participant with amputation at the residual limb patellar tendon site and contralateral limb lateral calf site, and a larger increase at the contralateral limb posterior calf site. Conversely, small biomarker decreases were observed all other sites, indicating increased tolerance to loading. Similar changes have also been observed during the application of different medical devices, including respiratory masks [38], cervical collars [28] and spinal boards [39]. Large variability was observed between participant responses with percentage change in IL- $1 \alpha / \mathrm{TP}$ ranging across all sites from -23 to $+470 \%$. Measurement site and environment have been shown to influence biomarker upregulation [37], although, in the present study, sampling was performed at a consistent location in a laboratory environment. Furthermore, the variability in biomarker expression is more likely a result of the differences between individuals and their respective physiological response to the applied loads. Similar variability in response has been reported in other studies, with distinct sub-populations of healthy cohorts demonstrating both high and low inflammatory responses [28, 29, 40]. This susceptibility to inflammation is worthy of further investigation, in conjunction with an examination of the temporal trends of inflammatory biomarker expression, regarding both pro-inflammatory and antagonistic cytokines [29, 39, 45]. IL-1RA/TP increased post-loading with a median percentage change of $81 \%, 30 \%$ and $89 \%$ at the patellar tendon, lateral calf and posterior calf respectively (Figure 6). The ratio of IL-1RA to IL-1 $\alpha$ is considered to reflect homeostatic regulation against inflammation $[46,47]$ and the comparable biomarker responses observed in this study indicate balanced inflammatory response, expected from healthy tissues within the test cohort. Monitoring cytokine response would enable assessment of applied loading, material and microclimate contributions. However, such an approach would be both difficult to achieve and expensive in the clinical setting, unless low cost devices can be developed offering point of care biomarker analyses.

The sample size of this current study was limited to 10 healthy participants and one example participant with amputation. The generalisation therefore is limited and further research is required with participants across a wide range of ages, activity levels and comorbidities to match the population with amputation. Furthermore, only static loading of low magnitudes was considered. Applied pressures were not randomised, as this would have required additional refractory periods in order to avoid effects from prior loading cases. Furthermore, with view to future tests on participants with amputation, a risk mitigation strategy would be to remove applied loading once a Category 3 response was reached, and this requires an incrementally increasing loading protocol.

The inflammatory response in this study is an accumulation of the pressures, time and materials. Investigation of the pressure-time relationship in higher resolution was beyond the scope of this study. However, Soetens et al [29] observed a significant change in inflammatory response upon loading and load removal at the sacrum, for both continuous and intermittent loading regimens. Cyclic loading may be more relevant to tissue damage during daily living activities with lower limb prosthetics. Further research is required to establish whether cyclic loading will increase the damage risk compared to static pressures [4] or, in some cases, provide a pumping mechanism to enhance vascular flow to the tissues [29]. In this study the participant's limb was in a supine position supported by foam cushions (Figure 1A) so vascular flow would have been less affected by gravity. However, studies utilising both seated and supine participant positions have also observed varying ischaemic responses 
between and within participants, studying both support surfaces and prosthetic devices $[19,21,23$, 24].

The viability of the residual limb tissues is of critical importance in prosthetic rehabilitation, particularly in the early stages post-amputation. In the clinical environment, effective tissue care can be achieved through regular skin assessments and advice provided by a multidisciplinary team. However, further evidence is required to establish a suitable measurement array and protocol to assess tissue tolerance and determine safe loading protocols, particularly in individuals with reduced sensory perception or diminished distal perfusion. Results demonstrate the potential of transcutaneous gas and biomarker measurement for early detection of precursors to tissue damage, with representative static prosthetic loading causing temporary local tissue ischaemia and an upregulation of inflammatory biomarkers released from the skin surface. Future studies are needed to establish appropriate noninvasive methods for use in clinical and community settings to monitor both the mechanical boundary conditions between the residual limb and socket, as well as the status of tissues. The presented array and protocol could be employed in a cohort of individuals with amputation to enhance knowledge on residuum tissue adaptation. Stratifying such cohorts will also help to determine factors that increase susceptibility to tissue damage at the residual limb-socket interface.

\section{Acknowledgements}

We would like to thank all of the individuals who participated in this study.

Funding: The authors would like to thank the following for their financial support:

- JLB: the University of Southampton's Institute for Life Sciences, and EPSRC Doctoral Training Program (ref EP/N509747/1)

- PRW, DLB, LEB: the EPSRC-NIHR "Medical Device and Vulnerable Skin Network" (ref $\mathrm{EP} / \mathrm{N} 02723 \mathrm{X} / 1$ ),

- ASD: the Royal Academy of Engineering, UK, (ref RF/130).

Ethics Committee approval for this protocol was granted by the University of Southampton (ERGO ID: 29696 and 41864).

Supporting data are openly available from the University of Southampton repository at http://dx.doi.org/10.5258/SOTON/D0910

\section{Disclosures}

None of the authors has any conflict of interest to declare. 


\section{References}

1. $\quad$ Oomens, C.W.J., et al., Pressure Induced Deep Tissue Injury Explained. Annals of Biomedical Engineering, 2015. 43(2): p. 297-305.

2. $\quad$ Bouten, C.V., et al., The etiology of pressure ulcers: skin deep or muscle bound? Arch Phys Med Rehabil, 2003. 84(4): p. 616-9.

3. Gray, R.J., D. Voegeli, and D.L. Bader, Features of lymphatic dysfunction in compressed skin tissues Implications in pressure ulcer aetiology. J Tissue Viability, 2016. 25(1): p. 26-31.

4. $\quad$ Peirce, S.M., T.C. Skalak, and G.T. Rodeheaver, Ischemia-reperfusion injury in chronic pressure ulcer formation: a skin model in the rat. Wound Repair Regen, 2000. 8(1): p. 68-76.

5. Meulenbelt, H.E., et al., Skin problems of the stump in lower limb amputees: 1. A clinical study. Acta Derm Venereol, 2011. 91(2): p. 173-7.

6. $\quad$ Coleman, S., et al., Developing a pressure ulcer risk factor minimum data set and risk assessment framework. J Adv Nurs, 2014. 70(10): p. 2339-52.

7. Lilja, M., P. Hoffmann, and T. Oberg, Morphological changes during early trans-tibial prosthetic fitting. Prosthet Orthot Int, 1998. 22(2): p. 115-22.

8. Pezzin, L.E., et al., Use and satisfaction with prosthetic limb devices and related services. Arch Phys Med Rehabil, 2004. 85(5): p. 723-9.

9. Sanders, J.E., et al., Residual limb fluid volume change and volume accommodation: Relationships to activity and self-report outcomes in people with trans-tibial amputation. Prosthet Orthot Int, 2018: p. 309364617752983.

10. Sanders, J.E. and B.S. Goldstein, Collagen fibril diameters increase and fibril densities decrease in skin subjected to repetitive compressive and shear stresses. J Biomech, 2001. 34(12): p. 1581-7.

11. Mueller, M.J. and K.S. Maluf, Tissue adaptation to physical stress: a proposed "Physical Stress Theory" to guide physical therapist practice, education, and research. Phys Ther, 2002. 82(4): p. 383-403.

12. Coleman, S., et al., A new pressure ulcer conceptual framework. J Adv Nurs, 2014. 70(10): p. 2222-34.

13. Hansen, C., et al., Incidence, severity, and impact of hyperhidrosis in people with lower-limb amputation. $\mathrm{J}$ Rehabil Res Dev, 2015. 52(1): p. 31-40.

14. Kottner, J., et al., Microclimate: A critical review in the context of pressure ulcer prevention. Clin Biomech (Bristol, Avon), 2018. 59: p. 62-70.

15. Zhang, M., et al., Clinical investigation of the pressure and shear stress on the trans-tibial stump with a prosthesis. Medical Engineering \& Physics, 1998. 20(3): p. 188-198.

16. Zachariah, S.G. and J.E. Sanders, Standing interface stresses as a predictor of walking interface stresses in the trans-tibial prosthesis. Prosthet Orthot Int, 2001. 25(1): p. 34-40.

17. Swanson, E.C., et al., Instrumented socket inserts for sensing interaction at the limb-socket interface. Med Eng Phys, 2018. 51: p. 111-118.

18. Bader, D.L. and P.R. Worsley, Technologies to monitor the health of loaded skin tissues. Biomed Eng Online, 2018. 17(1): p. 40.

19. Sangeorzan, B.J., et al., Circulatory and mechanical response of skin to loading. J Orthop Res, 1989. 7(3): p. 42531.

20. Vanross, E.R., S. Johnson, and C.A. Abbott, Effects of early mobilization on unhealed dysvascular transtibial amputation stumps: a clinical trial. Arch Phys Med Rehabil, 2009. 90(4): p. 610-7.

21. Rink, C.L., et al., Standardized Approach to Quantitatively Measure Residual Limb Skin Health in Individuals with Lower Limb Amputation. Adv Wound Care (New Rochelle), 2017. 6(7): p. 225-232.

22. Knight, S.L., et al., Establishing predictive indicators for the status of loaded soft tissues. Journal of Applied Physiology, 2001. 90(6): p. 2231-2237.

23. Worsley, P.R., et al., Monitoring the biomechanical and physiological effects of postural changes during leisure chair sitting. J Tissue Viability, 2018. 27(1): p. 16-22.

24. Chai, C.Y. and D.L. Bader, The physiological response of skin tissues to alternating support pressures in ablebodied subjects. Journal of the Mechanical Behavior of Biomedical Materials, 2013. 28: p. 427-435.

25. Uchi, H., et al., Cytokines and chemokines in the epidermis. J Dermatol Sci, 2000. 24 Suppl 1: p. S29-38.

26. Bronneberg, D., et al., Cytokine and chemokine release upon prolonged mechanical loading of the epidermis. Experimental Dermatology, 2007. 16(7): p. 567-573.

27. de Wert, L.A., et al., A new method to evaluate the effects of shear on the skin. Wound Repair and Regeneration, 2015. 23(6): p. 885-890.

28. Worsley, P.R., et al., Investigating the effects of cervical collar design and fit on the biomechanical and biomarker reaction at the skin. Med Devices (Auckl), 2018. 11: p. 87-94.

29. Soetens, J.F.J., et al., Investigating the influence of intermittent and continuous mechanical loading on skin through non-invasive sampling of IL-1alpha. J Tissue Viability, 2019. 
30. Cowley, K. and K. Vanoosthuyze, The biomechanics of blade shaving. Int J Cosmet Sci, 2016. 38 Suppl 1: p. $17-$ 23.

31. boso, User Instructions boso-profitest. 2012, Bosch + Sohn GmbH u. Co. KG.

32. Scott, H., et al., An evaluation of the Amputee Mobility Aid (AMA) early walking aid. Prosthet Orthot Int, 2000. 24(1): p. 39-46.

33. Gray, R.J., et al., Monitoring contractile dermal lymphatic activity following uniaxial mechanical loading. Medical Engineering \& Physics, 2016. 38(9): p. 895-903.

34. Unno, N., et al., A novel method of measuring human lymphatic pumping using indocyanine green fluorescence lymphography. J Vasc Surg, 2010. 52(4): p. 946-52.

35. Allen, V., et al., Accuracy of interface pressure measurement systems. J Biomed Eng, 1993. 15(4): p. 344-8.

36. Yigiter, K., G. Sener, and K. Bayar, Comparison of the effects of patellar tendon bearing and total surface bearing sockets on prosthetic fitting and rehabilitation. Prosthet Orthot Int, 2002. 26(3): p. 206-12.

37. Perkins, M.A., et al., A noninvasive method to assess skin irritation and compromised skin conditions using simple tape adsorption of molecular markers of inflammation. Skin Research and Technology, 2001. 7(4): p. 227-237.

38. Worsley, P.R., et al., Investigating the effects of strap tension during non-invasive ventilation mask application: a combined biomechanical and biomarker approach. Medical Devices-Evidence and Research, 2016. 9: p. 409-417.

39. Hemmes, B., et al., Cytokine ILlalpha and lactate as markers for tissue damage in spineboard immobilisation. A prospective, randomised open-label crossover trial. J Mech Behav Biomed Mater, 2017. 75: p. 82-88.

40. Bostan, L.E., et al., The influence of incontinence pads moisture at the loaded skin interface. J Tissue Viability, 2019. 28(3): p. 125-132.

41. Mirtaheri, P., et al., A review of the role of the partial pressure of carbon dioxide in mechanically loaded tissues: the canary in the cage singing in tune with the pressure ulcer mantra. Ann Biomed Eng, 2015. 43(2): p. 336-47.

42. Bader, D.L., Pressure Sores-Clinical Practice and Scientific Approach. 15. Effects of compressive loading regimens on tissue viability. 1990, Basingstoke, Hampshire: MACMILLAN PRESS Scientific \& Medical. 275.

43. Arsenault, K.A., et al., The use of transcutaneous oximetry to predict healing complications of lower limb amputations: a systematic review and meta-analysis. Eur J Vasc Endovasc Surg, 2012. 43(3): p. 329-36.

44. Wang, Z., et al., A systematic review and meta-analysis of tests to predict wound healing in diabetic foot. J Vasc Surg, 2016. 63(2 Suppl): p. 29S-36S.e1-2.

45. Cornelissen, L.H., et al., Cytokine Release in Tissue-Engineered Epidermal Equivalents After Prolonged Mechanical Loading. Epidermal Cells: Methods and Protocols, Second Edition, 2010. 585: p. 335-344.

46. Terui, T., et al., An increased ratio of interleukin-1 receptor antagonist to interleukin-1alpha in inflammatory skin diseases. Exp Dermatol, 1998. 7(6): p. 327-34.

47. Mee, J.B., et al., Counter-regulation of interleukin-1alpha (IL-1alpha) and IL-1 receptor antagonist in murine keratinocytes. J Invest Dermatol, 2005. 124(6): p. 1267-74. 


\section{Appendix}
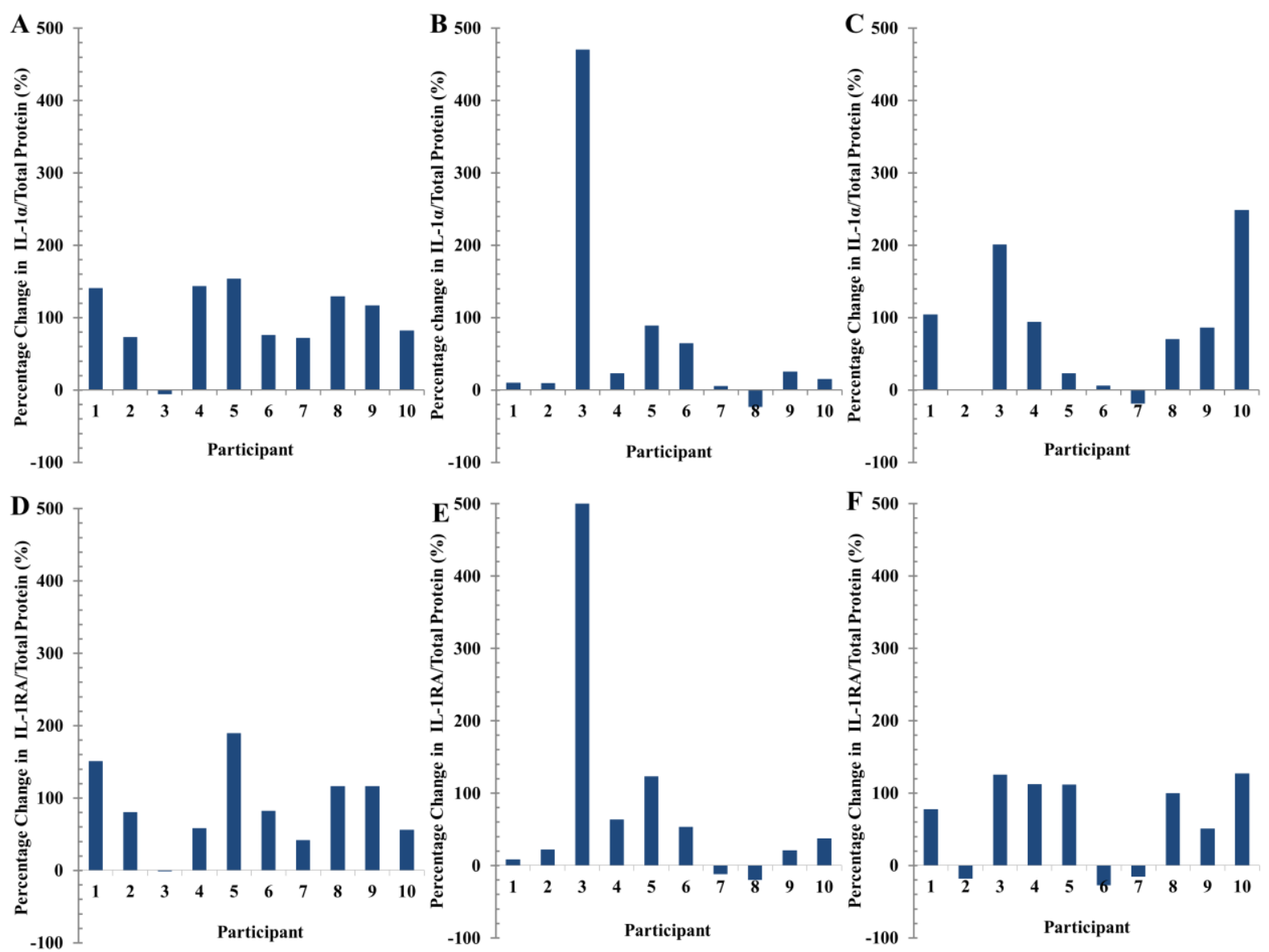

Figure 8: Percentage change in IL-1 $\alpha /$ Total protein ratio from baseline to post-cuff-loading up to $60 \mathrm{mmHg}$, at the patellar tendon (A), lateral calf $(B)$ and posterior calf $(C)$ and percentage change in IL-1RA/Total protein ratio from baseline to post-cuff-loading up to $60 \mathrm{mmHg}$, at the patellar tendon (D), lateral calf (E) and posterior calf (F) 